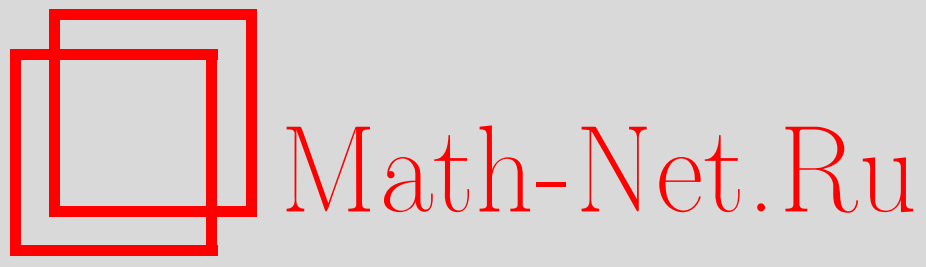

В. И. Арнольд, Топология алгебры: комбинаторика операции возведения в квадрат, Функи. анализ и его прил., 2003, том 37, выпуск 3, 20-35

DOI: https://doi.org/10.4213/faa155

Использование Общероссийского математического портала MathNet.Ru подразумевает, что вы прочитали и согласны с пользовательским соглашением

http://www.mathnet.ru/rus/agreement

Параметры загрузки:

IP : 52.205 .19 .152

26 апреля 2023 г., 13:13:34

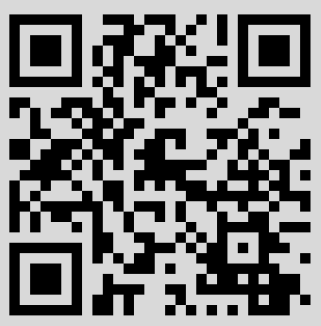


Функциональный анализ и его приложения

2003, т. 37 , вып. 3 , с. $20-35$

УДК $51+515+512+519.1+511+517.938$

\title{
Топология алгебры: комбинаторика операции возведения в квадрат*
}

\author{
(c) 2003. В. И. Арнольд
}

Объектом топологического изучения могут служить не только кривые и поверхности, но и алгебраические формулы. Топологическая структура простейших алгебраических теорий уже нетривиальна. Например, малая теорема Ферма (обобщенная Эйлером) есть утверждение о прямоугольности диаграммы Юнга перестановки взаимно простых с модулем вычетов при умножении их на один из этих вычетов. Ниже доказаны некоторые следствия подобных симметрий простейших алгебраических и теоретико-числовых конструкций, прежде всего операции возведения элементов групп или колец в квадрат.

Начинается эта теория с комбинаторики итераций любой операции, относящейся к общей теории множеств и логике больше, чем к алгебре.

\section{§1. Алгебра монад и топология их графов}

ОПРедЕЛЕниЕ 1. Монадой называется отображение конечного множества в себя. Граф монады имеет вершинами все элементы этого конечного множества, а ориентированные ребра соединяют каждый элемент с его образом.

Иными словами, граф монады - это произвольный конечный ориентированный граф, из каждой вершины которого выходит ровно одно ребро.

Итерации монады приводят любую вершину к циклу-аттрактору, как показывает следующая

Теорема 1. Каждая связная компонента графа монады представляет собой лес из ориентированных к корням корневых деревьев, корни которых соединены ориентированным ициклом (топологически окружностью) из ребер, соединяющих корни деревьев.

Число вершин цикла может быть равно 1, в этом случае вся компонента одно корневое дерево (см. примеры $A_{1}$ и $T_{4}$ ниже).

ДокАЗАТЕЛЬСтво. Траектория выходящего из любой вершины движения вдоль ориентированных ребер самопересекается вследствие конечности графа, поэтому цикл в компоненте есть.

Дерево точки цикла - это подграф, который имеет вершинами те вершины исходного графа, пути из которых впервые выходят на цикл в этой вершине. Этот подграф - дерево. Действительно, если бы в нем был цикл, то этот цикл был бы ориентирован (иначе из одной его вершины выходило бы два ребра).

Но два разных ориентированных циикла не могут лежать в одной компоненте связности графа монады, так как на связывающем их пути должна бы была быть вершина, из которой выходит больше одного ребра (лежащая, быть

\footnotetext{
*Частично поддержано РФФИ, грант 02-01-00655.
} 
может, на одном из циклов, что тоже невозможно, так как на циклах выходящие ребра направлены вдоль них).

Поэтому построенный подграф - дерево, ориентированное к лежащему на цикле корню, и теорема доказана.

Пример. Пусть $G$ - конечная группа, отображение «Фробениуса» $A: G \rightarrow G$ переводит каждый ее элемент $g$ в его квадрат: $A(g)=g^{2}$.

ОПредЕЛЕНИЕ 2. Это отображение $A$ назовем монадой группь $G$.

Графы монад простейших циклических групп (групп вычетов по модулю $n \leqslant 8$ ) изображены ниже. Операция в группе записана аддитивно, как сложение вычетов, надписанных около вершин графов, так что «возведение в квадрат» удваивает вычет. Выписанные справа обозначения графов определены в более общем виде ниже.

$$
\begin{aligned}
& \mathbb{Z}_{2}: \bigcirc^{0} \stackrel{1}{\longleftarrow}=A_{1} \text {; } \\
& \mathbb{Z}_{3}: \bigcap^{0} \stackrel{1}{\leftrightarrow}{ }^{2}=O_{1}+O_{2} \text {; }
\end{aligned}
$$

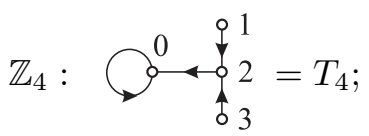

$$
\begin{aligned}
& \mathbb{Z}_{5}: \bigcirc^{0} \stackrel{1}{\leftrightarrow} \stackrel{2}{\leftrightarrow} \stackrel{3}{\leftrightarrow} \stackrel{4}{\longrightarrow}=O_{1}+O_{4} ;
\end{aligned}
$$

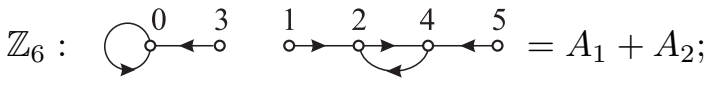

$$
\begin{aligned}
& \mathbb{Z}_{7}: \bigcap^{0} \stackrel{1}{\longleftrightarrow} \stackrel{2}{\longleftrightarrow} \stackrel{3}{\longleftrightarrow} \stackrel{6}{\longleftrightarrow} \stackrel{5}{\longleftrightarrow}=O_{1}+2 A_{3} ;
\end{aligned}
$$

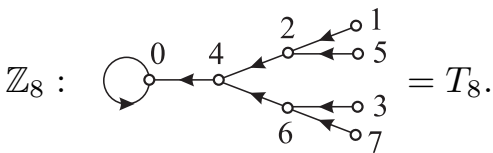

Здесь и ниже простейшие ориентированные графы обозначаются, как это указывает

ОПРЕДЕЛЕНИЕ 3. Символы $O_{n}, A_{n}, T_{m}, E_{n}$ означают следующие ориентированные графы:

$O_{n}=$ ориентированный цикл из $n$ вершин;

$A_{n}=$ связный граф из $2 n$ вершин, представляющий собой цикл длины $n$, оснащенный $n$ однореберными деревьями, входящими по одному в каждую из $n$ вершин цикла;

$T_{2^{n}}=$ корневое дерево с $2^{n}$ вершинами и $n$ этажами кроме корня, ветвящееся бинарно на этажах $1, \ldots, n-1$ (в каждую вершину такого этажа приходят ровно два ребра со следующего этажа); корень считается нулевым этажом, и в него тоже входят два ребра: одно - от него самого и одно - от единственной вершины первого этажа;

$E_{n}=$ корневое дерево с $n$ вершинами, из каждой из которых ребро ведет прямо в корень (так что $E_{2}=A_{1}=T_{2}$ ).

Применим теорему 1 к ситуации обобщенной Эйлером малой теоремы Ферма. 
ОПреДЕЛЕнИЕ 4. Группой Эйлера $Г(n)$ (где $n$ - натуральное число) называется мультипликативная группа, образованная всеми взаимно простыми с $n$ остатками от деления на $n$ (вычетами по модулю $n$ ), включая формально и тривиальный случай $\Gamma(1)=\{1\}$.

ПримеР. Список нетривиальных групп Эйлера начинается с групп

$$
\begin{gathered}
\Gamma(2)=\{1\}, \quad \Gamma(3) \approx \mathbb{Z}_{2}, \quad \Gamma(4) \approx \mathbb{Z}_{2}, \quad \Gamma(5) \approx \mathbb{Z}_{4}, \\
\Gamma(6) \approx \mathbb{Z}_{2}, \quad \Gamma(7) \approx \mathbb{Z}_{6}, \quad \Gamma(8) \approx \mathbb{Z}_{2} \times \mathbb{Z}_{2} .
\end{gathered}
$$

Описание всех групп Эйлера имеется, например, в школьной лекции [1].

Графы монад простейших групп Эйлера изображены ниже. Цифры у их вершин означают вычеты (элементы групп $\mathbb{Z}_{n}=\mathbb{Z} / n \mathbb{Z}$ ), но операция - умножение, а не сложение вычетов.

$$
\begin{aligned}
& \Gamma(2): \oint^{1}=O_{1} ; \\
& \Gamma(3): \overbrace{}^{1} \stackrel{2}{\leftarrow}=A_{1} \text {; } \\
& \Gamma(4): \bigcirc^{1} \overbrace{0}^{3}=A_{1} \text {; } \\
& \Gamma(5): \bigcap^{1} \mathfrak{o}_{3}^{2}=T_{4} ; \\
& \Gamma(6):
\end{aligned}
$$

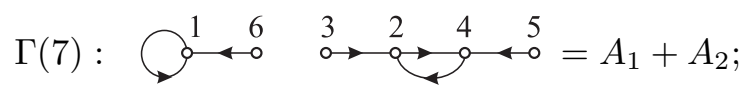

$$
\begin{aligned}
& \Gamma(8): \overbrace{07}^{1} \begin{array}{c}
03 \\
\leftarrow 05
\end{array}=D_{1} \text {. }
\end{aligned}
$$

ПримеР. Последний граф с 4 вершинами изображает 4 сравнения

$$
1^{2} \equiv 1(\bmod 8), \quad 3^{2} \equiv 1(\bmod 8), \quad 5^{2} \equiv 1(\bmod 8), \quad 7^{2} \equiv 1(\bmod 8),
$$

а предпоследний содержит сравнение $5^{2} \equiv 4(\bmod 7)$ (в своем крайне правом ребре) и еще пять других сравнений по модулю $7: 4^{2} \equiv 2$ и т. д.

Здесь использовано обозначение $D_{n}$ для $4 n$-вершинного графа, состоящего из цикла $O_{n}$ длины $n$, оснащенного в каждой своей вершине тремя подходящими к ней ребрами (образующими вместе с этой лежащей на цикле вершиной корневое дерево $\left.D_{1} \approx E_{4}\right)$.

Оснащение цикла $O_{n}$ этими деревьями является частным случаем следующей общей конструкции.

ОПреДЕЛЕНИЕ 5. Произведением $A * B$ монад $A$ и $B$, действующих на $X$ и на $Y$ соответственно, называется монада, действующая на прямом произведении $X \times Y$ покомпонентно:

$$
(A * B)(x, y)=(A x, B y) .
$$

Число элементов монады-произведения равно произведению чисел элементов монад-сомножителей. 
ОПРЕДЕЛЕНИЕ 6. Граф монады-произведения мы будем называть произведением графов сомножителей и обозначать знаком * операцию умножения и над графами:

$$
[г \operatorname{paф}(A * B)]=[г \operatorname{paq}(A)] *[г \operatorname{paф}(B)] .
$$

Пример. Имеют место тождества, разлагающие графы $A_{n}$ и $D_{n}$ на множители:

$$
A_{1} * O_{n}=A_{n}, \quad D_{1} * O_{n}=D_{n} .
$$

Это ассоциативное умножение «*» очевидно дистрибутивно относительно несвязного объединения «+» и может, как и объединение, считаться коммутативным (в силу естественных изоморфизмов $A+B \sim B+A, A * B \sim B * A$ ).

Умножение любого корневого дерева $X$ на $O_{n}$ оснащает $n$-цикл $O_{n}$ корневыми деревьями вида $X$ с корнями во всех точках цикла.

Мы исследуем ниже алгебру монад возведения в квадрат в коммутативных группах и, в частности, в группах Эйлера натуральных чисел, а также в симметрических группах.

\section{§2. Описание графов операции возведения в квадрат}

Начнем с монад и графов циклических групп, из которых все конечные коммутативные группы и их монады и графы получаются прямым перемножением.

ЛЕмма 1. Граф операции умножения на 2 (т.е. сложения элемента с самим собой) в аддитивной изиклической группе нечетного порядка разбивается на непересекающиеся цһикль $O_{n_{i}}$.

ДокАЗАТЕЛЬСтво. Если бы в какую-нибудь вершину вело два ребра, из $x$ и из $y$, то мы получили бы $2 x \equiv 2 y(\bmod (2 n+1))$ для циклической группы порядка $2 n+1$, т. е. $x \equiv y-$ одна и та же вершина.

Значит, в вершины цикла ведут пути графа только из точек этого же цикла, так что циклы и не пересекаются, и не оснащены лишними корневыми деревьями, что и доказывает лемму.

ЛЕмма 2. Граф операции умножения на 2 (сложения с собой) в аддитивной циклической группе вычетов по модулю $2^{n}$ есть бинарное корневое дерево $T_{2^{n}}$.

ДокАЗАТЕЛЬСтво. Мы уже видели, что это так для $n=1,2$ и 3 . В общем случае разделим все $2^{n}$ вычетов на две части: подгруппу из $2^{n-1}$ четных вычетов и остаток из $2^{n-1}$ нечетных вычетов.

Подгруппа четных вычетов циклическая (образуюшая - элемент 2). По индукции предполагаем, что граф удвоения в ней есть дерево $T_{2^{n-1}}$ и что верхний этаж в нем образуют $2^{n-2}$ не делящихся на 4 вычетов.

Докажем, что оставшиеся $2^{n-1}$ вершин (нечетные вычеты) образуют дополнительный $(n$-й $)$ этаж дерева $T_{2^{n}}$, т. е. что выходящие из них ребра ведут от ровно двух из них в каждую вершину верхнего (( $n-1)$-го) этажа предыдущего дерева, $T_{2^{n-1}}$.

А именно, этот верхний этаж образуют не делящиеся на 4 четные вычеты по модулю $2^{n}$. Удвоение нечетного вычета именно таково. Если два нечетных вычета $x$ и $y\left(\bmod 2^{n}\right)$ имеют общее удвоение, $2 x \equiv 2 y\left(\bmod 2^{n}\right)$, то $x \equiv y$ $\left(\bmod 2^{n-1}\right)$, т. е. вычет разности $x-y$ по модулю $2^{n}$ делится на $2^{n-1}$. Значит, он равен либо 0 , либо $2^{n-1}$, т. е. в вершину верхнего этажа дерева $T_{2^{n-1}}$ ведут два ребра из ровно двух нечетных вычетов по модулю $2^{n}$, что и доказывает лемму. 
Коммутативная группа представляет собой прямое произведение циклических групп. Поэтому из лемм 1 и 2 вытекает

Теорема 2. Монады всех конечных коммутативных групп представляют собой суммы произведений нескольких монад видов $O_{n} u T_{2^{m}}$.

Пример. Монада группы Эйлера $\Gamma(125) \approx \mathbb{Z}_{100}$ есть сумма трех слагаемых:

$$
\left[\text { монада }\left(\mathbb{Z}_{100}\right)\right]=T_{4}+\left(T_{4} * O_{4}\right)+\left(T_{4} * O_{20}\right) .
$$

Для получения графа операции возведения в квадрат всех 125 вычетов по модулю 125 нужно прибавить еще одно слагаемое: корневое 25-вершинное дерево с четырьмя приходящими к корню ребрами, два из которых начинаются в общем конце десятка ребер (каждое).

\section{§3. Таблица умножения графов}

Лемма 3. Произведение изиклических монад $O_{m}$ и $O_{n}$ есть сумма одинаковых циклических монад, а именно

$$
O_{m} * O_{n}=d O_{c},
$$

где число слагаемых $d=(m, n)-$ наибольший общий делитель периодов $m$ u $n$, а $c=m n / d-$ их наименьшее общее кратное.

Точно так же вычисляется и произведение нескольких циклических монад: это повторенная d раз циклическая монада $O_{c}$, где $c$ - наименьшее общее кратное порядков сомножителей, а dc равно произведению этих порядков.

Примеры. Имеют место тождества $\mathrm{O}_{2} * \mathrm{O}_{2}=2 \mathrm{O}_{2}, \mathrm{O}_{3} * \mathrm{O}_{6}=3 \mathrm{O}_{6}, \mathrm{O}_{6} * O_{15}=$ $3 \mathrm{O}_{30}$.

ДокАЗАТЕЛЬСтво. Проекции орбиты каждого элемента-произведения, последовательно возводимого в квадрат $t$ раз, имеют наименьшие периоды $t=m$ для проекции на $O_{m}$ и $t=n$ для проекции на $O_{n}$. Поэтому наименьший общий период в $O_{m} * O_{n}$ есть наименьшее общее кратное, $c$, чисел $m$ и $n$. А так как число вершин графа $O_{m} * O_{n}$ есть $m n$, то число орбит есть $m n / c=d$.

Полезно отметить, что $O_{2} * O_{2 k}=2 O_{2 k}, O_{2} * O_{2 k+1}=O_{4 k+2}, O_{3} * O_{5}=O_{15}$. Для большего числа сомножителей утверждение следует из доказанного выше для двух сомножителей.

СледствиЕ. Имеют место соотношения

$$
O_{m} * A_{n}=d A_{c}, \quad A_{m} * A_{n}=d D_{c} .
$$

Первое следует из леммы 3 ввиду соотношения $A_{n}=A_{1} * O_{n}$, а второе - еще и из доказанных выше соотношений $D_{n}=D_{1} * O_{n}, A_{1} * A_{1}=D_{1}$.

Для вычисления всевозможных произведений графов и монад $D_{n}$ (на $O_{m}, A_{m}$, $\left.D_{m}\right)$ полезно отметить, что $A_{1}^{k}$ есть граф $E_{2^{k}}$ : корневое дерево из $2^{k}$ вершин, ребра из каждой из которых сразу ведут к корню (например, $E_{2}=A_{1}, E_{4}=D_{1}$ ).

Действительно, каждый элемент группы $\left(\mathbb{Z}_{2}\right)^{k}$ становится нулем при сложении с самим собой (которое и есть «возведение в квадрат» при аддитивной записи группы).

Например, имеют место тождества (графов и монад)

$$
D_{1}^{2}=A_{1} * A_{1} * A_{1} * A_{1}=E_{16}, \quad D_{m} * D_{n}=d E_{16} O_{c} .
$$


Вычислим граф группы Эйлера $\Gamma(125)$, равной $\mathbb{Z}_{100} \approx \mathbb{Z}_{4} \times \mathbb{Z}_{25}$. Начнем с вычисления графов для групп-сомножителей.

Граф удвоений элементов ииклической группы нечетного порядка $\mathbb{Z}_{25}$ разбивается на циикль длин 20, 1 u 4, так как орбитами удвоения вычетов по модулю 25 являются

$$
0,\{1,2,4,8,16,7,14,3,6,12,(24 \sim-1),-2, \ldots,(-12 \sim 13)\},\{5,10,20,15\} .
$$

Стало быть, монада удвоений циклической группы $\mathbb{Z}_{25}$ есть сумма трех слагаемых $O_{1}+O_{4}+O_{20}$. С другой стороны, граф монады удвоений циклической группы $\mathbb{Z}_{4}$ есть дерево $T_{4}$. Стало быть, для произведения мы получаем представление

$$
\left[г \text { раф }\left(\mathbb{Z}_{100}\right)\right]=T_{4}+\left(T_{4} * O_{4}\right)+\left(T_{4} * O_{20}\right) .
$$

В явном виде эти три слагаемых (с указанием вычетов вершин) таковы:
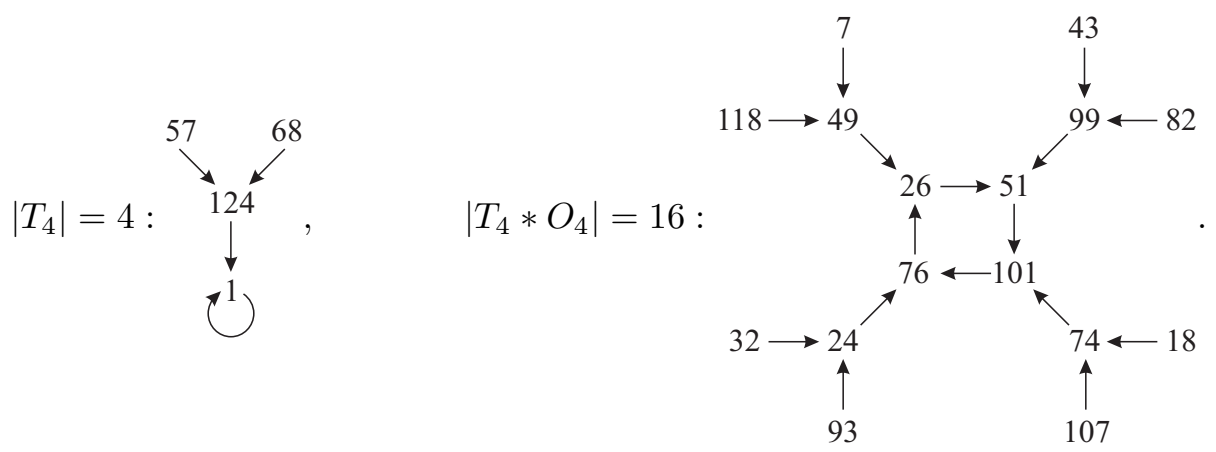

Это значит, например, что $26^{2} \equiv 51(\bmod 100)$ и т. д. Особенно долго и чревато ошибками вычисление самой большой компоненты графа (топологию которой лучше найти заранее):

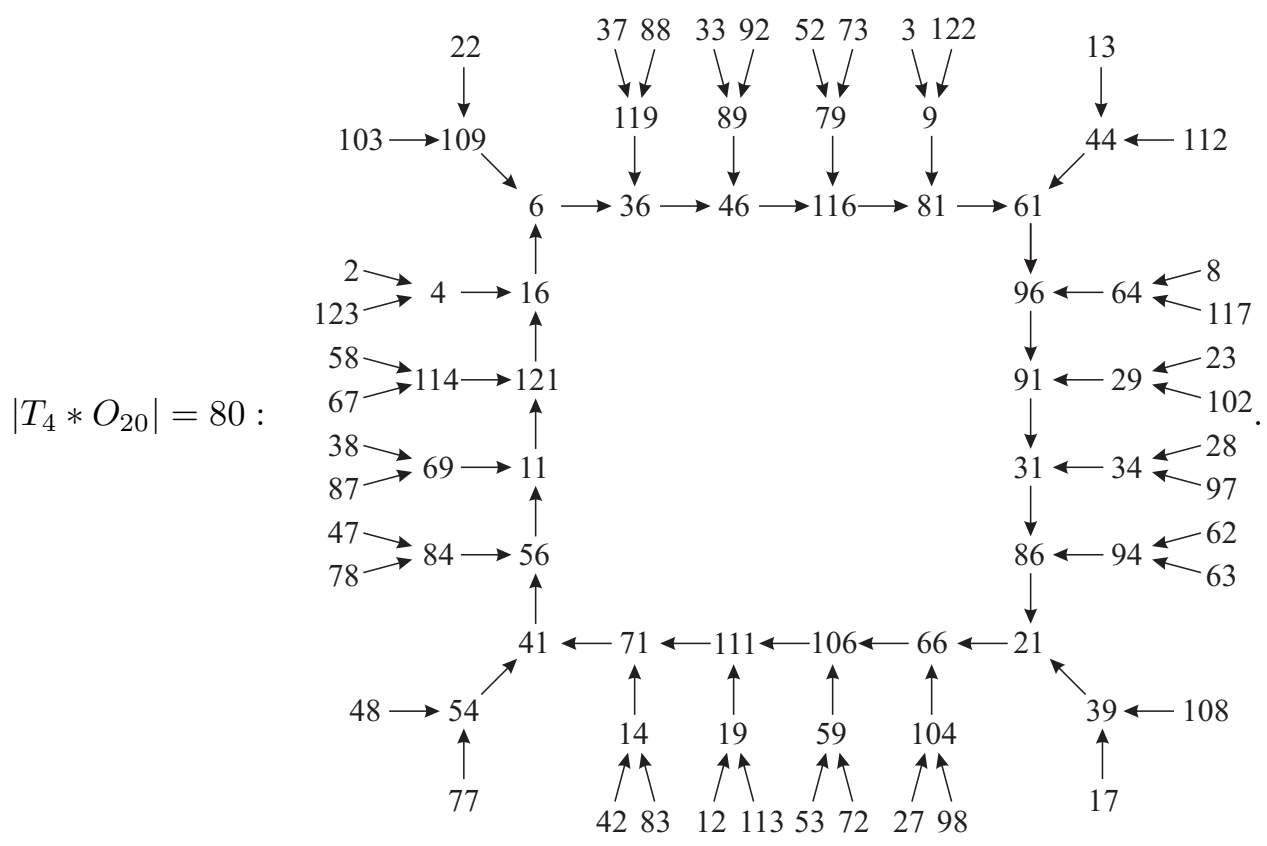


Таблицы квадратичных вычетов в учебниках должны бы были выглядеть именно так.

Симметрии всех этих графов являются далекими обобщениями малой теоремы Ферма (эти связи подробно описаны в [1-3]).

Теорема 2 (\$2) показывает, что никаких более сложных узоров, кроме произведений нескольких деревьев $T_{2^{m_{i}}}$ и ичиклов $O_{n}$, в графах монад коммуmaтuвных грyпn не встречается: граф конечной коммутативной группы есть несвязное объединение циклов, оснащенных произведениями бинарных деревьев, т. е. объединение графов вида $O_{n} * \prod T_{2} m_{i}$. Вычисление числа слагаемых и значений чисел $m_{i}$ и $n$ через порядки циклических сомножителей требует, однако, исследования периода $T$ операции удвоения (вычетов по фиксированному модулю). Это исследование, начатое Ферма (для простого модуля) и Эйлером (для составного) продолжено в [1-3], но даже средняя асимптотика периода $T(n)$ прогрессии $\left\{2^{t}(\bmod n)\right\}$ не доказана (эксперименты подсказывают, по-видимому, степенной в среднем рост периода $T$ с модулем $n$ ). Не вычислено явно и число компонент графа для операции удвоения в $\Gamma(n)$.

\section{§4. Таблицы графов удвоений в циклических группах}

Ниже перечислены графы монад $x \mapsto 2 x$ в аддитивных циклических группах $\mathbb{Z}_{n}$ вычетов по модулю $n \leqslant 20$.

\begin{tabular}{|c|l||r|l||l|l||c|l|}
\hline$n$ & граф удвоений & $n$ & граф удвоений & $n$ & граф удвоений & $n$ & граф удвоений \\
\hline 2 & $A_{1}$ & 7 & $O_{1}+2 O_{3}$ & 12 & $T_{4}+\left(T_{4} * O_{2}\right)$ & 17 & $O_{1}+2 O_{8}$ \\
3 & $O_{1}+O_{2}$ & 8 & $T_{8}$ & 13 & $O_{1}+O_{12}$ & 18 & $A_{1}+A_{2}+A_{6}$ \\
4 & $T_{4}$ & 9 & $O_{1}+O_{2}+O_{6}$ & 14 & $A_{1}+2 A_{3}$ & 19 & $O_{1}+O_{18}$ \\
5 & $O_{1}+O_{4}$ & 10 & $A_{1}+A_{4}$ & 15 & $O_{1}+O_{2}+3 O_{4}$ & 20 & $T_{4}+\left(T_{4} * O_{4}\right)$ \\
6 & $A_{1}+A_{2}$ & 11 & $O_{1}+O_{10}$ & 16 & $T_{16}$ & & \\
\hline
\end{tabular}

Для нечетного простого числа $n=p$ граф удвоений имеет вид $O_{1}+N O_{T(p)}$, где $T(p)-$ наименьший период геометрической прогрессии $\left\{2^{t}(\bmod p)\right\}$.

Доказательства этих фактов изложенными выше методами несложны. Например, $\mathbb{Z}_{15}=\mathbb{Z}_{3} \times \mathbb{Z}_{5}$, поэтому граф (монада) группы $\mathbb{Z}_{15}$ есть произведение графов (монад) сомножителей:

$$
\begin{aligned}
{\left[г \operatorname{paф}\left(\mathbb{Z}_{15}\right)\right] } & =\left(O_{1}+O_{2}\right)\left(O_{1}+O_{4}\right)=O_{1} O_{1}+O_{1} O_{4}+O_{2} O_{1}+O_{2} O_{4} \\
& =O_{1}+O_{4}+O_{2}+2 O_{4}=O_{1}+O_{2}+3 O_{4} .
\end{aligned}
$$

Мы использовали, что $\mathrm{O}_{2} \mathrm{O}_{4}=2 \mathrm{O}_{4}$ по лемме $3 \S 3$ (так как наибольший общий делитель чисел 2 и 4 есть 2 , а наименьшее их общее кратное есть 4 ).

Встречающееся при $n=12$ и при $n=20$ произведение $T_{4} * O_{k}$ легко вычисляется: этот связный граф из $4 k$ вершин состоит из $k$ корневых деревьев $T_{4}$ с соединенными в цикл длины $k$ своими корнями. В примере, вычисляющем граф для $\mathbb{Z}_{12}$, дерево $T_{4} * O_{2}$ имеет следующий вид (с указанием восьми вычетов $(\bmod 12)$, являющихся его вершинами):

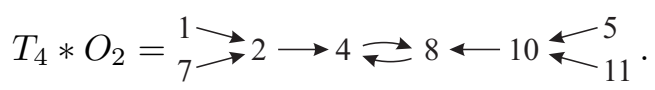

Это значит, например, что $7+7 \equiv 2(12), 8+8 \equiv 4(12)$. 
Укажу заодно простейшие графы $T_{4} * A_{k}$ : они имеют, как легко сосчитать, вид
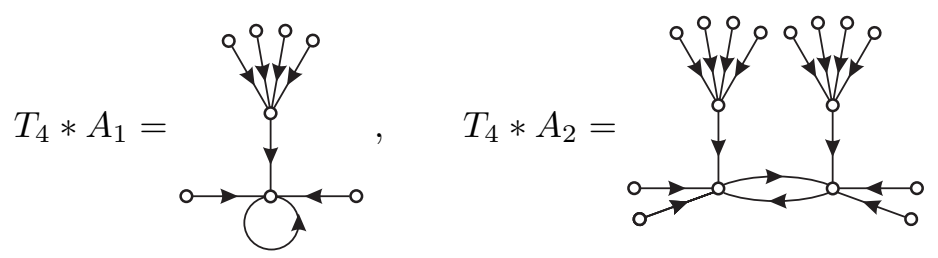

с одним циклом длины $k$, двумя подходящими к каждой точке цикла простыми ребрами и еще одним, оснащающим каждую вершину корневым деревом (имеющим одну вершину на первом этаже и четыре на втором). Умножение деревьев обсуждается ниже в $\$ 7$.

Вычисления в алгебре графов гораздо проще, чем прямое вычисление квадратов вычетов. Например, для $n=20$ вычисления таковы:

$\left[г\right.$ раф удвоений в $\left.\mathbb{Z}_{20}\right]=\left[\right.$ граф удвоений в $\left.\mathbb{Z}_{4}\right] *\left[\right.$ граф удвоений в $\left.\mathbb{Z}_{5}\right]$

$$
=T_{4} *\left(O_{1}+O_{4}\right)=T_{4}+\left(T_{4} * O_{4}\right) .
$$

Мы использовали тождество $T_{4} * O_{1}=T_{4}$ (тривиальный граф $O_{1}$ с одной вершиной является единицей нашего умножения).

\section{§5. Графы возведения в квадрат в группах Эйлера}

Ниже перечислены графы монад $x \mapsto x^{2}$ в группах Эйлера $\Gamma(n)$ (мультипликативных группах взаимно простых с $n$ вычетов по модулю $n$ ) и в содержащих их кольцах $\mathbb{Z}_{n}$ (для $\left.n \leqslant 20\right)$.

Мы воспользуемся сведениями из [1]: цикличностью групп Эйлера $\Gamma(n)$, когда число $n$ простое или степень нечетного простого числа,

$$
\Gamma\left(p^{a}\right) \approx \mathbb{Z}_{\varphi\left(p^{a}\right)}, \quad \text { где } \varphi\left(p^{a}\right)=(p-1) p^{a-1},
$$

а также соотношением мультипликативности

$$
\Gamma(m n)=\Gamma(m) \times \Gamma(n)
$$

при взаимно простых сомножителях $m$ и $n$. Кроме того,

$$
\Gamma\left(2^{n}\right)=\mathbb{Z}_{2} \times \mathbb{Z}_{2^{n-2}},
$$

так что вычисление графа возведения в квадрат в группе Эйлера $\Gamma(n)$ всегда сводится к перемножению графов «удвоения» в циклических сомножителях группы Эйлера, рассматриваемых как аддитивные группы вычетов.

Используя таблицу $\$ 4$, мы находим следующий список графов операции возведения в квадрат в группах Эйлера $\Gamma(n)$ :

\begin{tabular}{|l|l||r|l||l|l||l|l|}
\hline$n$ & $\begin{array}{l}\text { граф возведе- } \\
\text { ния в квадрат }\end{array}$ & $n$ & $\begin{array}{l}\text { граф возведе- } \\
\text { ния в квадрат }\end{array}$ & $n$ & $\begin{array}{l}\text { граф возведе- } \\
\text { ния в квадрат }\end{array}$ & $n$ & $\begin{array}{l}\text { граф возведе- } \\
\text { ния в квадрат }\end{array}$ \\
\hline 2 & $O_{1}$ & 7 & $A_{1}+A_{2}$ & 12 & $D_{1}$ & 17 & $T_{16}$ \\
3 & $A_{1}$ & 8 & $D_{1}$ & 13 & $T_{4}+\left(T_{4} * O_{2}\right)$ & 18 & $A_{1}+A_{2}$ \\
4 & $A_{1}$ & 9 & $A_{1}+A_{2}$ & 14 & $A_{1}+A_{2}$ & 19 & $A_{1}+A_{2}+A_{6}$ \\
5 & $T_{4}$ & 10 & $T_{4}$ & 15 & $T_{4} * A_{1}$ & 20 & $T_{4} * A_{1}$ \\
6 & $A_{1}$ & 11 & $A_{1}+A_{4}$ & 16 & $T_{4} * A_{1}$ & & \\
\hline
\end{tabular}


Например, $\Gamma(15)=\Gamma(3) \times \Gamma(5)$, поэтому граф возведения в квадрат в группе $\Gamma(15)$ есть произведение графов возведения в квадрат в группах $Г(3)$ и $\Gamma(5)$, т. е. графов, равных $A_{1}$ и $T_{4}$ соответственно. Можно воспользоваться и тем, что $\Gamma(3) \approx \mathbb{Z}_{2}, \Gamma(5) \approx \mathbb{Z}_{4}$, так что достаточно перемножить графы удвоений в аддитивных группах $\mathbb{Z}_{2}$ и $\mathbb{Z}_{4}$, указанные в таблице $\$ 4$.

Точно так же, граф возведения в квадрат в циклической группе $\Gamma(13) \approx \mathbb{Z}_{12}$ есть граф операции удвоения в аддитивной группе вычетов по модулю 12 , указанный в таблице §4. Все результаты приведенной выше таблицы графов операции возведения в квадрат получаются подобными вычислениями произведений из результатов $\S 4$.

\section{§6. Возведение в квадрат в кольце вычетов}

«Расширяя» группу Эйлера $\Gamma(n) \subset \mathbb{Z}_{n}$ до всего кольца вычетов по модулю $n$ (которое уже не является мультипликативной группой), мы можем и здесь рассмотреть операцию возведения вычета в квадрат. Граф этой монады содержит граф возведения в квадрат в группе Эйлера $\Gamma(n)$ в виде поднабора компонент связности, так как квадрат вычета не может быть взаимно простым с модулем, если сам вычет таковым не был (и не может перестать быть взаимно простым с модулем при возведении в квадрат).

Поэтому для описания графа операции возведения в квадрат в кольце вычетов $\mathbb{Z}_{n}$ достаточно добавить к графу этой операции в группе Эйлера $\Gamma(n)$ еще некоторый (не связанный ребрами с ним) дополнительный граф $\Delta$.

Эта конструкция похожа на формулу Эйлера

$$
n=\sum \varphi(d) \quad(d=1, \ldots, n-\text { делитель числа } n),
$$

в которой слагаемое $\varphi(n)$ соответствует вкладу графа группы Эйлера $\Gamma(n)$, а остальные слагаемые - вкладу дополнительного графа $\Delta$.

Дополнительные графы $\Delta$ для операции возведения в квадрат вычетов по модулю $n$ имеют (при $n \leqslant 20$ ) следующий вид:

\begin{tabular}{|r|l||r|l||r|l|||l|l|}
\hline$n$ & \multicolumn{1}{|c||}{$\Delta(n)$} & $n$ & \multicolumn{1}{c|}{$\Delta(n)$} & $n$ & \multicolumn{1}{c|}{$\Delta(n)$} & $n$ & \multicolumn{1}{c|}{$\Delta(n)$} \\
\hline 2 & $O_{1}$ & 7 & $O_{1}$ & 12 & $2 A_{1}+D_{1}$ & 17 & $O_{1}$ \\
3 & $O_{1}$ & 8 & $T_{4}$ & 13 & $O_{1}$ & 18 & $A_{1}+A_{2}+2 E_{3}$ \\
4 & $A_{1}$ & 9 & $E_{3}$ & 14 & $2 O_{1}+A_{1}+A_{2}$ & 19 & $O_{1}$ \\
5 & $O_{1}$ & 10 & $2 O_{1}+T_{4}$ & 15 & $O_{1}+A_{1}+T_{4}$ & 20 & $2 A_{1}+\left(T_{4} * A_{1}\right)$ \\
6 & $2 O_{1}+A_{1}$ & 11 & $O_{1}$ & 16 & $T_{4} * A_{1}$ & & \\
\hline
\end{tabular}

Знаком $E_{n}$ здесь обозначено корневое дерево с $n$ вершинами, из каждой из которых ведет сразу в корень ребро (так что $\left.E_{2}=A_{1}, E_{4}=D_{1}\right)$. Доказательство сформулированной этой таблицей теоремы несложно.

В случае простого значения модуля $n$ группа Эйлера состоит из всех вычетов, кроме нуля, так что к графу возведения в квадрат в группе Эйлера добавляется только одна эта точка $O_{1}$. В нее не ведет ни одно другое ребро, так как квадрат ненулевого вычета по простому модулю - ненулевой вычет.

В других случаях приходится исследовать действие возведения в квадрат на не взаимно простых с модулем вычетах. Например, для модуля 6 мы получаем 
граф возведения вычетов в квадрат из четырех компонент (две типа $O_{1}$ и две типа $\left.A_{1}\right)$ :

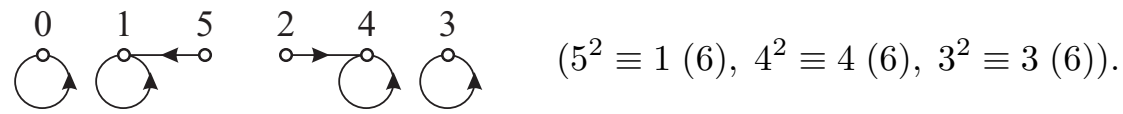

Группу Эйлера $\Gamma(5) \approx \mathbb{Z}_{2}$ составляет компонента $A_{1}=\{1,5\}$, а остальные три компоненты доставляют добавочный граф $\Delta=2 O_{1}+A_{1}$.

\section{§7. Произведения корневых деревьев}

Произведение монад, графы которых являются корневыми деревьями, также имеет графом корневое дерево, которое мы будем называть произведением двух исходных деревьев.

Вершинами произведения являются пары вершин сомножителей, и если в графе $X$ из $x$ выходит ребро, ведущее в $a$, а из $y$ в графе $Y-$ ведущее в $b$, то из вершины $(x, y)$ дерева $X * Y$ ребро выходит в $(a, b)$. Цикл в произведении $X * Y$ проектируется в циклы в $X$ и в $Y$, поэтому произведение корневых деревьев является корневым деревом.

ОПРЕДЕЛЕНИЕ 7. Ранг $r_{i}(X)$ корневого дерева - это число его вершин $i$-го этажа, т. е. отстоящих от корня на $i$ ребер. Например, $r_{0}(X)=1, r_{1}\left(A_{1}\right)=1$, $r_{1}\left(D_{1}\right)=3, r_{1}\left(E_{m}\right)=m-1, r_{i}\left(T_{2^{n}}\right)=2^{i-1}$ при $0<i \leqslant n$.

При перемножении корневых деревьев ранги вершин расстояния $i$ ведут себя так:

$$
r_{k}(X * Y)=\sum r_{i}(X) r_{j}(Y)
$$

где $\max (i, j)=k$.

Это следует из того, что путь, ведущий в корень произведения, проектируется в пару путей, ведущих в корни сомножителей, а пара путей, ведущих в корни сомножителей, всегда является парой проекций определяемого ими пути, ведущего в корень в произведении.

Это соображение позволяет легко перемножать, например, деревья $T_{2^{n}}$ :

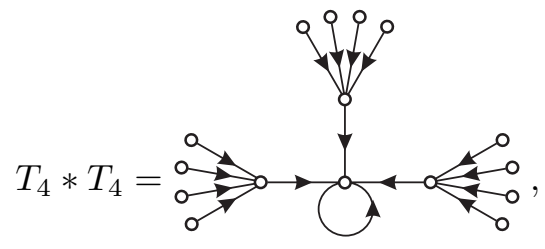

так как таблица произведений рангов, $r_{i} r_{j}$, есть

\begin{tabular}{|l|l|l|}
\hline 1 & 1 & 2 \\
\cline { 1 - 1 } 1 & 1 & 2 \\
\cline { 1 - 2 } 2 & 2 & 4 \\
\hline
\end{tabular}

и поэтому $r_{k}\left(T_{4} * T_{4}\right)=(1,3,12)(k=0,1,2)$ (суммирование по отмеченным уголкам матрицы произведений рангов).

Точно так же мы получаем ранги других произведений деревьев $T_{2^{n}}$.

Теорема 3. Ранги квадрата бинарного корневого дерева суть

$$
r_{k}\left(T_{2^{n}} * T_{2^{n}}\right)=3 \cdot 4^{k-1} \quad \text { npu } k=1, \ldots, n .
$$


Если заменить второй сомножитель бо́льшим бинарньм деревом, $T_{2^{m}}$, где $m>n$, то последовательность рангов продолжится степенями двойки:

$$
\left(r_{n+1}, \ldots, r_{m}\right)=\left(4^{n}, 2 \cdot 4^{n}, 4 \cdot 4^{n}, \ldots, 2^{m-1}\right) .
$$

Произведения $T_{8} * T_{2}\left(r_{i}=1,3,4,8\right)$ и $T_{8} * T_{4}\left(r_{i}=1,3,12,16\right)$ выглядят так:

\begin{tabular}{|l|l|l|l|}
\hline 1 & 1 & 2 & 4 \\
\hline 1 & 1 & 2 & 4 \\
\hline
\end{tabular}

$T_{8} * T_{2}=$

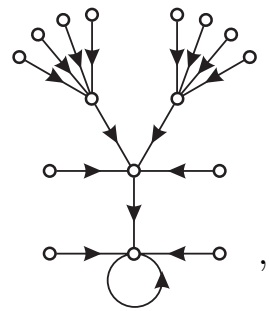

\begin{tabular}{|l|l|l|l|}
\hline 1 & 1 & 2 & 4 \\
\hline 1 & 1 & 2 & 4 \\
\hline 2 & 2 & 4 & 8 \\
\hline
\end{tabular}

$T_{8} * T_{4}=$

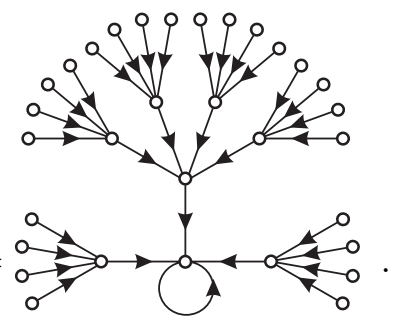

Число ребер графа-произведения, приходящих в вершину-произведение, равно произведению чисел ребер графов-сомножителей, приводящих в умножаемые вершины в сомножителях. Число $R_{n}$ (не обязательно бинарных) корневых деревьев с $n$ (не считая корня) вершинами растет с числом вершин так:

\begin{tabular}{|c|cccccccc|}
\hline$n$ & 0 & 1 & 2 & 3 & 4 & 5 & 6 & 7 \\
\hline$R_{n}$ & 1 & 1 & 2 & 4 & 9 & 20 & 48 & 114 \\
\hline
\end{tabular}

Отсюда следует, что большинство корневых деревьев просто (не представляется в виде произведения меньших деревьев). Я не знаю ни как растет число простых корневых деревьев, ни единственно ли разложение корневого дерева на простые множители. Малость $R_{n}$ по сравнению с факториалом указывает на изобилие автоморфизмов меньших корневых деревьев.

Перечисление корневых деревьев близко к рассматривавшейся, по словам Плутарха, Гиппархом [4] задаче о том, сколько сложных предложений можно составить из $n$ простых (для $n=10$ число сложных предложений равно 103049) и к теореме Кэли о числе корневых деревьев с $n$ занумерованными некорневыми вершинами (число таких деревьев равно $(n+1)^{n-1}$, степени отображения ЛяшкоЛоенги особенности $A_{n}$, т. е. $1,3,16,125, \ldots$ при $\left.n=1,2, \ldots\right)$. Сравнение асимптотик этих чисел и чисел $R_{n}$ доставляет информацию о числе автоморфизмов случайного корневого дерева.

\section{§8. Графы возведения в квадрат в симметрических группах}

Граф операции возведения в квадрат в группе $S(n)$ перестановок $n$ элементов можно вычислять при помощи диаграмм Юнга перестановок, составляющих эту группу. Диаграмма Юнга перестановки определяет диаграмму Юнга ее квадрата. Ответы получаются следующие (ребра графов ведут от диаграмм Юнга перестановок к диаграммам Юнга их квадратов).

$S(2) \approx \mathbb{Z}_{2}$, поэтому граф есть

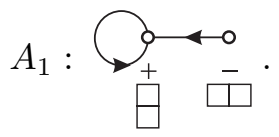

У каждой вершины графа указана диаграмма Юнга той перестановки, которой является эта вершина. Знак минус у диаграммы Юнга указывает на нечетность соответствующей перестановки. 
Группа $S(3)$ симметрий треугольника имеет 3 элемента второго порядка и содержит кроме них еще подгруппу вращений $\mathbb{Z}_{3}$, поэтому граф возведения в ней в квадрат состоит из двух компонент: $D_{1}+O_{2}$,

$$
D_{1}: \boxminus \bigcirc
$$

Группа $S(4)$ разрешима и имеет нормальным делителем подгруппу $S^{+}(4)$ из 12 четных перестановок. Графы возведения в квадрат в обеих этих группах состоят каждый из пяти компонент связности.

$\left[\right.$ Граф группы $\left.S^{+}(4)\right]=D_{1}+4 O_{2}$,

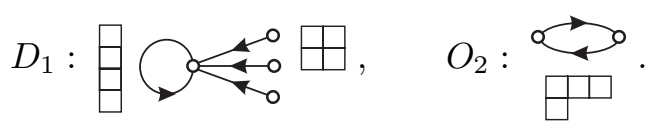

Граф всей группы $S(4)$ имеет вместо $D_{1}$ компоненту, являющуюся корневым деревом с рангами $(1,9,6)$ :

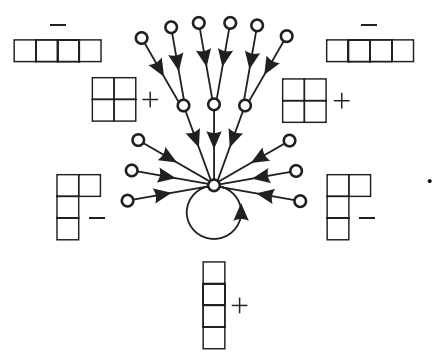

Группа $S^{+}(5)$ состоит из 60 четных перестановок пяти элементов. Ее граф состоит из семнадцати компонент,

$\left[г\right.$ раф группы $\left.S^{+}(5)\right]=10 O_{2}+6 O_{4}+E_{16}$,

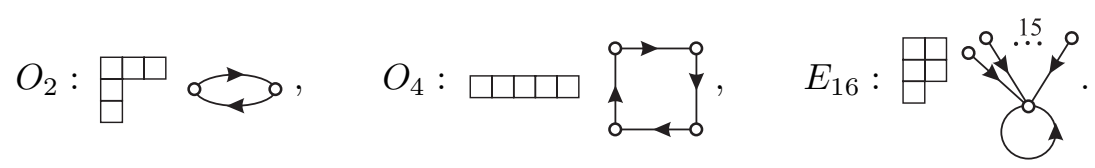

Группа $S(5)$ всех 120 перестановок пяти элементов имеет граф тоже из 17 компонент,

$[$ граф группы $S(5)]=10 A_{2}+6 O_{4}+B$,

где корневое дерево $B$ имеет ранги $(1,25,30)$ :

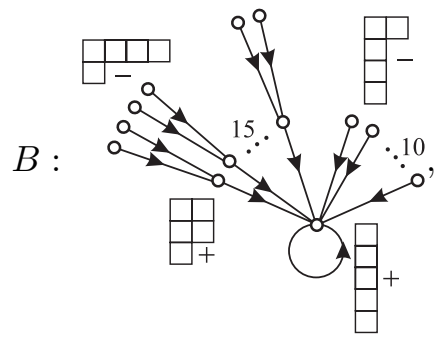

$A_{2}$ :
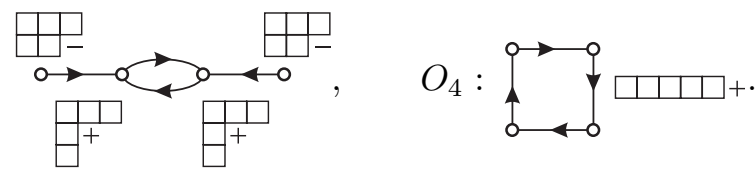
Группа $S^{+}(6)$ состоит из 360 четных перестановок шести элементов. Ее граф состоит из 77 компонент:

$$
\text { [граф группы } \left.S^{+}(6)\right]=36 O_{4}+40 O_{2}+C^{+},
$$

где корневое дерево $C^{+}$имеет ранги $(1,45,90)$ : диаграммы Юнга вершин этих компонент указаны ниже:
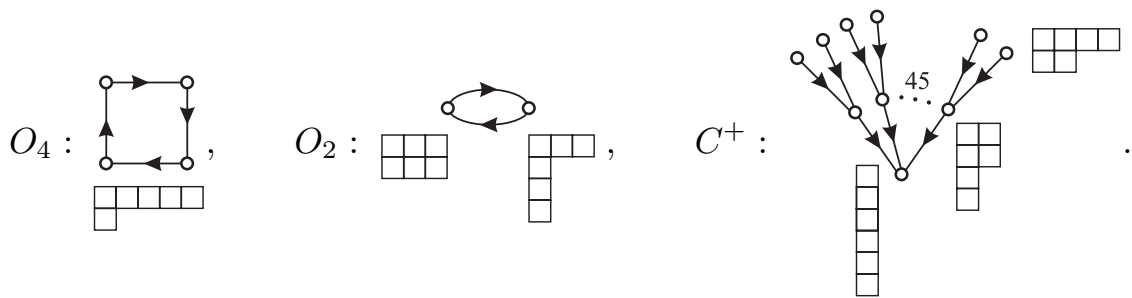

С учетом 360 нечетных перестановок компонент тоже 77 :

[граф группы $S(6)]=36 O_{4}+40 D_{2}+C$.

Половина из сорока компонент вида $D_{2}$ образована циклическими перестановками и их квадратами,

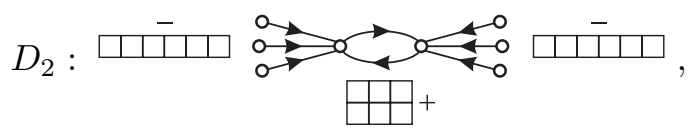

а вторая половина - перестановками с циклами длин $(3,2,1)$ и их квадратами (длины циклов которых $(3,1,1,1))$ :

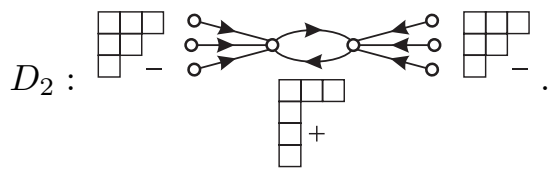

Корневое дерево $C$ имеет ранги $(1,75,180)$. Диаграммы Юнга перестановок, являющихся его вершинами, таковы:

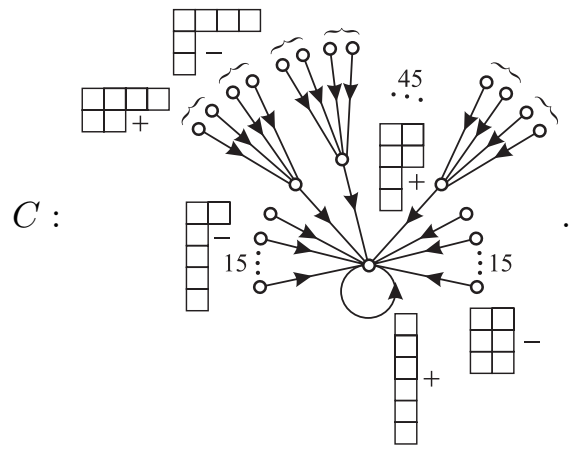

Группа $S^{+}(7)$ состоит из 2520 четных перестановок семи элементов. Ее граф имеет 542 связные компоненты, а именно

[граф группы $\left.S^{+}(7)\right]=240 O_{3}+126 O_{4}+140 O_{2}+35 D_{2}+Y$, 
где корневое дерево $Y$ имеет ранги $(1,105,630)$. Вид компонент графа:
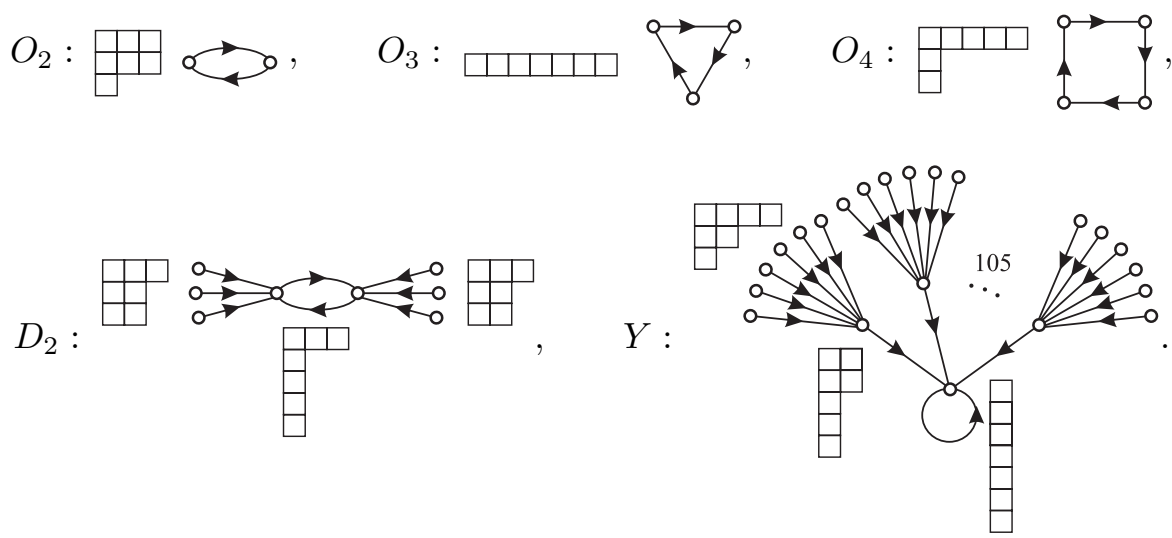

Полный граф всей группы 5040 перестановок семи элементов имеет тоже 542 компоненты:

$$
[г \text { раф группы } S(7)]=240 O_{3}+124 \widetilde{O}_{4}+140 \widetilde{O}_{2}+35 \widetilde{D}_{2}+\widetilde{Y} \text {. }
$$

Индекс в обозначении указывает порядок цикла, оснащением которого является компонента. Волной обозначены здесь изображенные ниже расширения компонент графа $S^{+}(7)$. Корневое дерево $\widetilde{Y}$ изображено ниже и имеет ранги $(1,231,840)$. Имеют место топологические тождества $\widetilde{O}_{2} \sim D_{2}, \widetilde{O}_{4} \sim A_{4}$.

Перечень диаграмм Юнга перестановок, являющихся вершинами расширенных компонент графа операции возведения в квадрат в группе $S(7)$, содержит все 15 диаграмм Юнга площади 7:
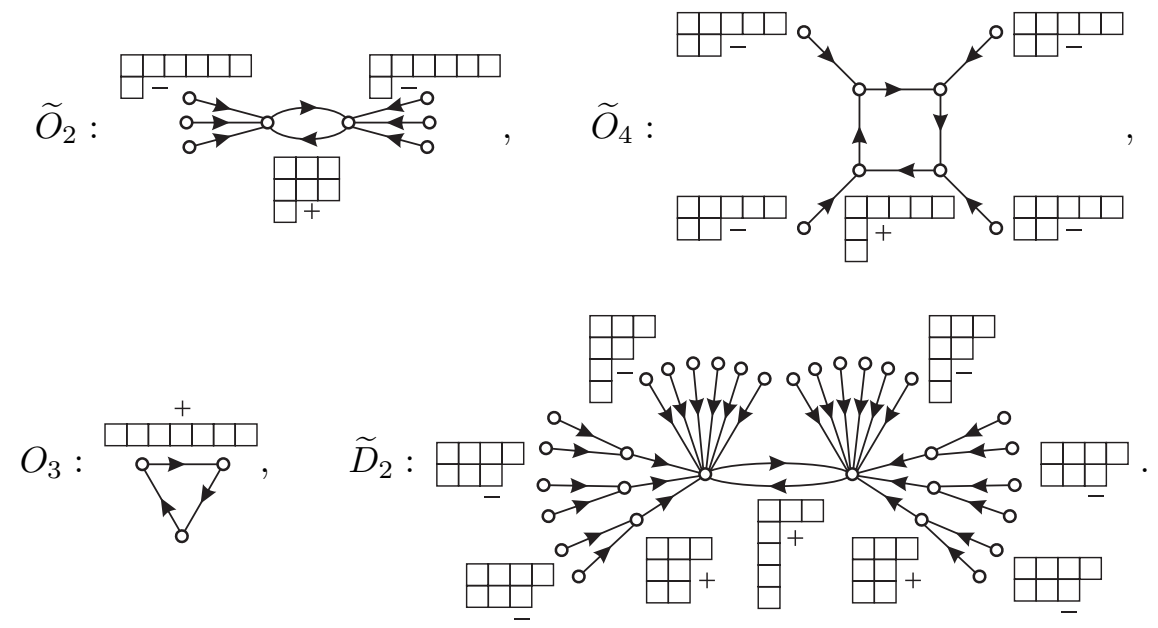
Компонента тождественной перестановки имеет вид следующего дерева $\widetilde{Y}$ :

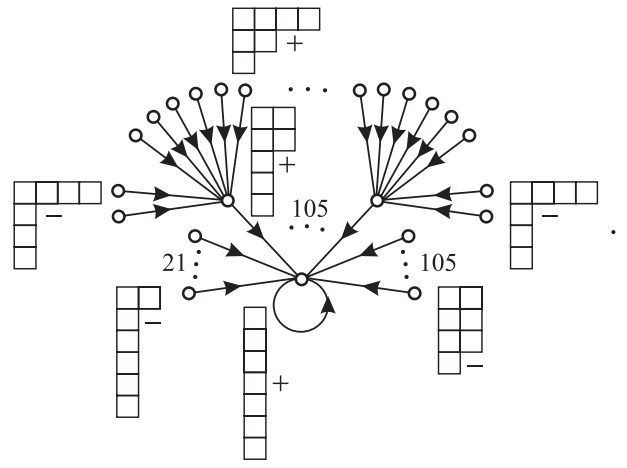

Например, шесть ребер, ведущих от перестановок с циклами длин $(4,2,1)$ к одной из 105 перестановок с циклами длин $(2,2,1,1,1)$, означают следующие факты. Перестановка с циклами длин $(4,2,1)$ может быть записана в

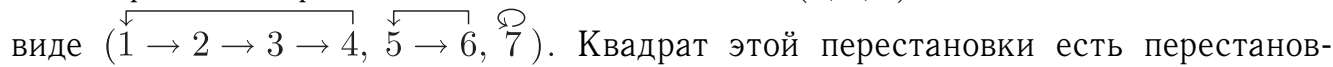
ка $\left(\begin{array}{lllllll}1 & 2 & 3 & 4 & 5 & 6 & 7 \\ 3 & 4 & 1 & 2 & 5 & 6 & 7\end{array}\right)$ с циклами длин $(2,2,1,1,1)$. Докажем, что такой же квадрат имеют ровно 6 (из 630) перестановок с ичиклами длин $(4,2,1)$. В исходной перестановке цикл $(1,2,3,4)$ можно, не меняя квадрата, заменить только на $(1,4,3,2)$, а транспозицию $(5,6)$ - только на $(5,7)$ или $(6,7)$. Два варианта цикла длины 4 и три варианта цикла длины 2 порождают (единственные) $3 \cdot 2=6$ комбинаций, имеющих циклы длин $(4,2,1)$ и фиксированный квадрат.

Эти шесть перестановок с циклами длин $(4,2,1)$ и соединены со своим общим квадратом (с циклами длин $(2,2,1,1,1))$ на изображении дерева $\widetilde{Y}$, в его верхней части.

ТЕорема 4. Каждая связная компонента графа возведения в квадрат группь $S(n)$ перестановок $n$ элементов представляет собой однородно оснащенный циикл, т.е. граф вида $O_{k} * T$, где $T$ - корневое дерево. Это верно и для подгруппь $S^{+}(n)$ четных перестановок.

ДокАЗАТЕЛЬСтво. Если перестановка $x$ принадлежит циклу длины $k>1$, то она имеет нечетный порядок $N$, так как $k$-кратное возведение $x$ в квадрат превращает эту перестановку в свою четную степень $x^{M}$, и условие замыкания цикла $x^{M}=x$ означает нечетность ее порядка $N=M-1$.

ЛЕмма. Перестановка нечетной степени сопряжена своему квадрату.

ДокАЗАТЕЛЬство. Все циклы такой перестановки имеют нечетные длины. Квадрат циклической перестановки $z$ нечетной длины $m$ - тоже циклическая перестановка, так как все элементы $\left(z^{2}\right)^{t} a(t=1, \ldots, m)$ различны, иначе перестановка $z$ имела бы цикл четной длины, меньшей $2 m$.

Поэтому циклическая перестановка нечетной длины $m$ сопряжена своему квадрату, а значит, это верно и для любой перестановки нечетной степени, и лемма доказана.

Построенное по перестановке $x$ сопряжение группы $S(n)$ переводит граф операции возведения в квадрат в себя, причем его точка $x$ переходит в $x^{2}$, т.е. сдвигается на одно место вдоль цикла. Компонента этого цикла вся переходит при построенном сопряжении в себя, с поворотом цикла на одно ребро. 
Граф любой монады имеет компонентами оснащенные деревьями циклы-аттракторы (см. теорему $\$ 1$ ). Построенная симметрия компоненты доказывает однородность этого оснащения (одинаковость оснащающих все вершины цикла деревьев).

Для подгруппы четных перестановок лемма не верна (например, циклические перестановки $1 \rightarrow 2 \rightarrow 3$ и $1 \rightarrow 3 \rightarrow 2$ степени 3 не сопряжены в ней). Но и теорему, и доказательство, можно перенести на этот случай, используя сопряженность не в группе четных, а в группе всех перестановок (доставляющую не внутренний, а внешний автоморфизм группы $S^{+}$). Этот автоморфизм тоже определяет симметрию графа, доказывающую однородность оснащения цикла.

Значения $k$ длин циклов компонент графов и ранги $r_{i}$ деревьев $T$, соответствующих этим компонентам, приведены для первых групп перестановок $(S)$ и четных перестановок $\left(S^{+}\right) n$ элементов в следующей таблице.

\begin{tabular}{|l|c|cc|cc|ccc|cccc|ccccc|}
\hline$n$ & 2 & \multicolumn{3}{|c|}{3} & \multicolumn{2}{|c|}{4} & \multicolumn{3}{|c|}{5} & \multicolumn{3}{|c|}{6} & \multicolumn{5}{|c|}{7} \\
\hline$k$ & 1 & 1 & 2 & 1 & 2 & 1 & 2 & 4 & 1 & 2 & 4 & 1 & 2 & 2 & 3 & 4 \\
\hline & 1 & 1 & 1 & 1 & 1 & 1 & 1 & 1 & 1 & 1 & 1 & 1 & 1 & 1 & 1 & 1 \\
$r \mid S$ & 1 & 3 & & 9 & & 25 & 1 & & 75 & 3 & & 231 & 3 & 9 & & 1 \\
& & & & 6 & & 30 & & & 180 & & & 840 & & 6 & & \\
\hline & 1 & 1 & 1 & 1 & 1 & 1 & 1 & 1 & 1 & 1 & 1 & 1 & 1 & 1 & 1 & 1 \\
$r \mid S^{+}$ & & & 3 & & 1 & & & 45 & & & 105 & & & & \\
& & & & & & & & 90 & & & 630 & & & & \\
\hline
\end{tabular}

Однородность оснащения циклов компонент деревьями сохраняется для всех конечных групп.

Было бы интересно исследовать поведение топологии графа группы при косом умножении: как связаны графы операции возведения в квадрат для групп $G, E$ и $H$ точной последовательности $1 \rightarrow H \rightarrow E \rightarrow G \rightarrow 1$ ? Вопрос интересен уже для двулистных накрытий, когда $H=\mathbb{Z}_{2}$, и для подгрупп индекса 2 , когда $G=\mathbb{Z}_{2}$, как для $S^{+} \rightarrow S \rightarrow S / S^{+}$.

Перекос топологии графа косого произведения групп может геометрически кодировать информацию о степени алгебраической перекошенности «произведения» $E$ групп $G$ и $H$.

\section{ЛИТЕРАТУРА}

1. Арнольд В. И. Группы Эйлера и арифметика геометрических прогрессий. МЦНМО, 2003.

2. Арнольд В. И. Динамическая система Ферма-Эйлера и статистика арифметики геометрических прогрессий. Функц. анализ и его прил., 37, вып. 1, 1-18 (2003).

3. Arnold V. Ergodic and arithmetic properties of geometrical progression's dynamics and of its orbits. Moscow Mathematical Journal, 4, 1-20 (2004).

4. Плутарх. Застольные беседы. Перевод Я. М. Боревского. М., 1987, VIII.9, с. 157.

5. Арнольд В. И. Топология и статистика формул арифметики. УМН, 58, вып. 4 (354), $1-26$ (2003).

Математический институт им. В. А. Стеклова,

CEREMADE, Universitè Paris-Dauphine

Поступило в редакцию 23 октября 2002 г. 